\title{
Effects of Dietary Extruded Linseed (Linum usitatissimum) and Oregano (Origanum vulgare) on Growth Traits, Carcass Composition and Meat Quality of Grigia di Potenza Suckling Kids
}

\author{
Simona Tarricone ${ }^{1}$, Francesco Giannico ${ }^{2}$, Marco Ragni ${ }^{1 *}$, Maria Antonietta Colonna ${ }^{1}$, Pasqua Rotondi ${ }^{1}$, Carlo \\ Cosentino $^{3}$, Alireza Seidavi ${ }^{4}$, Vincenzo Tufarelli ${ }^{* *}$ and Vito Laudadio ${ }^{5}$ \\ ${ }^{1}$ Department of Agro-Environmental and Territorial Sciences, University of Bari 'Aldo Moro', 70125 Bari, Italy \\ ${ }^{2}$ Department of Veterinary Medicine, University of Bari 'Aldo Moro', 70010 Valenzano, Bari, Italy \\ ${ }^{3}$ School of Agricultural, Forestry, Food and Environmental Sciences, University of Basilicata, 85100 Potenza, Italy \\ ${ }^{4}$ Department of Animal Science, Rasht Branch, Islamic Azad University, Rasht 41335-3516, Iran \\ ${ }^{5}$ Department of DETO, Section of Veterinary Science and Animal Production, University of Bari 'Aldo Moro', 70010 Bari, Italy \\ For correspondence: marco.ragni@uniba.it \\ Received 20 October 2020; Accepted 26 December 2020; Published 16 April 2021
}

\begin{abstract}
The aim of this trial was to compare the influence of supplementing diets with extruded linseed and oregano on growth parameters and meat qualitative traits in Grigia di Potenza breed suckling kids. Twenty-four male kids, exclusively fed milk from their dams, were assigned to the following diets: C) group control fed without any supplement; L) group fed control feed containing 3\% extruded linseed (Linum usitatissimum L.); and LO) group fed control diet with $0.6 \%$ dried oregano (Origanum vulgare) and 3\% extruded linseed. Growth performance as well as slaughtering traits and meat cuts of kids were not significantly influenced $(P>0.05)$ by dietary treatments. Conversely, kids in linseed group reported the lower $(P<0.05)$ percentage of dissectible fat in leg and loin. The meat from Longissimus lumborum and Semimembranosus muscles of kids in linseed diet had the lowest $(P<0.05)$ cooking loss percentage, whereas the proximate chemical composition of both meat muscles did not vary among treatments $(P>0.05)$. The experimental diets partially modulated the kid meat fatty acid composition in both muscles, where feeding linseed and oregano improved $(P<0.05)$ the content of DPA and reduced MUFA. Based on the current findings, it can be concluded that linseed and oregano supplementation can be used in goat diet as no significant detrimental effects on productive performance and meat quality of suckling kids were observed. (C) 2021 Friends Science Publishers
\end{abstract}

Keywords: Diet; Extruded linseed; Goat; Meat quality; Oregano; Suckling kids

\section{Introduction}

Small ruminants are the dominant species of domestic livestock animals in the South of Italy, including the Basilicata region. Generally, in this area sheep and goats are mainly farmed under extensive or semi extensive conditions based on natural pastures. In these marginal areas, small ruminant rearing system is performed traditionally using local breeds due to their excellent flexibility to the environmental conditions and utilization of the existing feeding resources (Selvaggi and Tufarelli 2012). Goat autochthonous breeds are typical dual-purpose, developed to produce the unweaned slaughter kids at an early age, so that farmer can continue long milking goats to process milk into traditional cheeses.

The Grigia di Potenza goat is the most common autochthonous breed reared in Matera province, Basilicata region of Italy (www.capre.it). This goat breed has an average size, with a weight of about $50-70 \mathrm{~kg}$, having fine and light head, with big hanging ears, usually horned, and this goat can produce up to 300-350 litre of milk per lactation. Livestock species graze on natural pastures and receive additional feeds based on concentrate mixture composed in function of productivity level and nutrient requirements. New feeding strategies can include the use of natural feed additive to increase the polyunsaturated fatty acids (PUFA) content of meat. In the last decade several attempts have been made to increase the PUFA content in meat from livestock species, including diet raw linseed and linseed oil supplementation in lambs (Giannico et al. 2009; Colonna et al. 2011; Toteda et al. 2011; Facciolongo et al. 2018), kids (Rotondi et al. 2018), steers (Ragni et al. 2014; Tarricone et al. 2019a) and pigs (Tarricone et al. 2019b). However, achieving this enrichment in ruminants is

To cite this paper: Tarricone S, F Giannico, M Ragni, MA Colonna, P Rotondi, C Cosentino, A Seidavi, V Tufarelli, V Laudadio (2021). Effects of dietary extruded linseed (Linum usitatissimum) and oregano (Origanum vulgare) on growth traits, carcass composition and meat quality of Grigia di Potenza suckling kids. Intl J Agric Biol 25:1147-1152 
challenging owing to the biohydrogenation of fatty acids which occurs in rumen (Lunn and Theobald 2006). The supplementation of ruminants' diets with natural antioxidants is considered an effective strategy for modulating and improving fatty acid composition of meat (Cimmino et al. 2018). Natural antioxidants such as oregano, sage, thyme and rosemary are well accepted by consumers, because of they are considered safe and healthy (Naveena et al. 2016). The antioxidant activity in oregano is mainly attributed to carvacrol and thymol (Tufarelli et al. 2017). Trials using oregano (Origanum vulgare) supplied to lamb diet have assessed its efficacy in enhancing meat quality as well its sensory parameters (Simitzis et al. 2008); nevertheless, to date limited data are available on the dietary effects of oregano to the kids (Rotondi et al. 2018). The relevance and high quality of kid meat produced in Basilicata region of southern Italy were studied by many researchers (Ragni et al. 2015; Colonna et al. 2020). However, to the best our knowledge, data on qualitative traits of Grigia di Potenza kids are to date not available.

Therefore, the aim of this research was to compare the influence of supplementing goat diet with oregano and extruded linseed on productive traits and quality of meat in suckling kids belonging the Grigia di Potenza goat breed.

\section{Materials and Methods}

\section{Animal management and diet}

The feeding trial was conducted out at the farm "Masseria Pian del Camino" located in Albano di Lucania, Basilicata region, Italy $\left(40^{\circ} 36^{\prime} 03.7^{\prime \prime} \mathrm{N}, 16^{\circ} 03^{\prime} 00.1^{\prime \prime} \mathrm{E} ; 899 \mathrm{~m}\right.$ above sea level) and involved twenty-four male Grigia di Potenza breed kids, born as twins. Kids were managed following the traditional local rearing method for Grigia di Potenza goat breed as kids fed exclusively milk their from dams (about up to 21 days of age). So, kids were assigned to the following treatments: C) group control fed without any supplement; L) group fed control feed containing 3\% extruded linseed (Linum usitatissimum L.); and LO) group fed control diet with $0.6 \%$ dried oregano (Origanum vulgare) and 3\% extruded linseed. Supplied concentrates were isoenergetic and isoproteic, and to cover the kids requirements as for NRC (2007) indications (Tables 1 and 2). During the day the dams grazed and received ad libitum hay and concentrate (about $600 \mathrm{~g}$ per head daily) during the evening at housing. Maternal goat milk samples were collected twice daily for nutrient and fatty acid analysis (Table 2).

Animals were housed individually in pens (space allowance: $8000 \mathrm{~cm}^{2}$ per head) with ad libitum water, and rearing temperature conditions in pens varied from $7-15^{\circ} \mathrm{C}$. At sixty days kids were slaughtered, following exsanguination, by an official veterinarian after a fasting of $12 \mathrm{~h}$, having kids free water, and registering the kids weight. The weight of hot carcass, fleece, skin, pluck, and gut were recorded. Each carcass was hung and chilled at refrigeration temperature of $4^{\circ} \mathrm{C}$ (relative humidity: $80-82 \%$ ) for 24 hours and immediately weighed. The carcass dressing percentage was also calculated. The carcass refrigerated were split into two halves by midline; the right side was divided into cuts The right-side carcass was cut into wholesale cuts and separately weighed following the indications of Sarvar et al. (2009). The kids leg and loin were conducted from slaughterhouse to lab at $4^{\circ} \mathrm{C}$. The two cuts of meat, except tail fat, were stored under refrigeration for additional $24 \mathrm{~h}$, and separated into the three parts (lean, dissectible fat and bone) and each weight recorded (Tufarelli et al. 2011).

\section{Feed and milk chemical composition}

Samples of concentrates were collected every 15 days and mixed to get a final pool for each treatment, then analysed to assess the nutritional characteristics. Sample was ground in a hammer mill using a 1-mm screen and analysed (AOAC 2000). Goat milk samples were collected twice daily (morning and evening), stored at $4^{\circ} \mathrm{C}$ and transported immediately to lab and analysed for total protein, lactose and fat (Milko Scan 133B; Foss Electric, DK-3400, Denmark).

\section{Meat parameters}

Values of meat $\mathrm{pH}$ were determined on kid muscles (Longissimus lumborum and Semimembranosus) at slaughter and after $24 \mathrm{~h}$ at $4^{\circ} \mathrm{C}$, by a $\mathrm{pH}$-meter, and at two-point calibration. On both muscles meat colour and tenderness were determined. The colour of meat, in terms of lightness $L^{*}$, redness $a^{*}$, and yellowness $b^{*}$, was assessed using a spectrophotometer (Tarricone et al. 2019a). The readings $(n=3)$ were done for individual samples placing the instrument on diverse areas of meat as reported by Šicklep and Čandek-Potokar (2007). Samples of the three meat (about $1.25 \mathrm{~cm}$ thick and diameter) from each muscle were tested to determine the Warner-Bratzler Shear (WBS) as for Tarricone et al. (2019a). To assess meat cooking loss, samples of about $5 \mathrm{~cm}$ thick were cut from the muscles and weighed before and after cooking using a oven ventilated at $165^{\circ} \mathrm{C}$, until reaching an internal temperature of $75^{\circ} \mathrm{C}$ in meat. To determine the meat nutritional composition (AOAC 2000), sub-samples of kids meat muscles were analysed for fat content (Folch et al. 1957). Concentrate feeds were used for FA profile based on the method as above; also, the FA were reported as percent of total methylated FA.

\section{Statistical analysis}

Data were analyzed using the GLM procedure of SAS application package (SAS 2004). The differences among means were assessed using the LSD procedure. The effect of initial kid body weight as a linear covariate was omitted from model due to its not significant effect. The significance level was set at $P<0.05$. 
Oregano and Extruded Linseed in Kid Diet / Intl J Agric Biol, Vol 25, No 5, 2021

Table 1: Composition of the experimental diets fed to goats

\begin{tabular}{llll}
\hline Ingredients (\% as-fed basis) & \multicolumn{3}{c}{ Diets $^{1}$} \\
\cline { 2 - 4 } & $\mathrm{C}$ & $\mathrm{L}$ & $\mathrm{LO}$ \\
\hline Corn & 31.00 & 31.00 & 30.40 \\
Faba bean & 10.00 & 8.50 & 8.50 \\
Wheat bran & 10.00 & 10.00 & 10.00 \\
Barley & 9.00 & 9.00 & 9.00 \\
Wheat flour shorts & 9.00 & 9.00 & 9.00 \\
Sunflower meal & 8.00 & 7.50 & 7.50 \\
Dehulled soybean & 6.00 & 6.00 & 6.00 \\
Sugar beet pulp & 6.00 & 6.00 & 6.00 \\
Soybean hulls & 4.00 & 4.00 & 4.00 \\
Molasses & 3.00 & 3.00 & 3.00 \\
Vitamin-mineral premix & 3.00 & 3.00 & 3.00 \\
Soybean oil & 1.00 & - & - \\
Extruded linseed & - & 3.00 & 3.00 \\
Oregano (dried inflorescences) & - & - & 0.60 \\
\hline${ }^{1}$ C, control feed; L, control feed $+3 \%$ extruded linseed; LO, control feed + 3\% \\
extruded linseed + 0.6\% oregano
\end{tabular}

Table 2: Chemical and fatty acid composition of diets and maternal goat milk

\begin{tabular}{|c|c|c|c|c|}
\hline \multirow[t]{2}{*}{ Item } & \multicolumn{3}{|c|}{ Diets $^{1}$} & \multirow[t]{2}{*}{ Milk } \\
\hline & $\mathrm{C}$ & $\mathrm{L}$ & $\mathrm{LO}$ & \\
\hline \multicolumn{5}{|c|}{ Chemical composition (\% on DM basis) } \\
\hline Crude protein & 16.79 & 16.68 & 17.23 & 3.46 \\
\hline Ether extract & 4.59 & 5.58 & 6.16 & 4.73 \\
\hline Ash & 9.09 & 9.07 & 9.01 & 0.86 \\
\hline Lactose & - & - & - & 4.35 \\
\hline Crude fiber & 15.18 & 13.43 & 14.97 & - \\
\hline $\mathrm{NDF}^{2}$ & 33.85 & 36.00 & 36.55 & - \\
\hline $\mathrm{ADF}^{2}$ & 10.93 & 11.68 & 11.95 & - \\
\hline $\mathrm{ADL}^{2}$ & 2.64 & 2.91 & 3.07 & - \\
\hline \multicolumn{5}{|c|}{ Fatty acid composition (\% FA methyl esters) } \\
\hline C6:0 & - & - & - & 1.18 \\
\hline C8:0 & - & - & - & 2.17 \\
\hline C10:0 & - & - & - & 9.15 \\
\hline C12:0 & 0.95 & - & - & 4.42 \\
\hline $\mathrm{C} 14: 0$ & 0.94 & - & - & 9.47 \\
\hline C16:0 & 9.17 & 7.64 & 7.63 & 24.70 \\
\hline $\mathrm{C} 17: 0$ & 0.66 & - & - & 0.63 \\
\hline C18:0 & 1.15 & 3.69 & 4.03 & 10.94 \\
\hline C18:1 n-9, cis 9 & 17.91 & 18.83 & 17.95 & 22.32 \\
\hline C18:2n-6 & 39.16 & 22.03 & 20.58 & 3.04 \\
\hline C18:3 n-3 & 4.54 & 31.06 & 30.70 & 0.88 \\
\hline C20:4 n-6 & 0.21 & - & - & 0.32 \\
\hline$C 22: 5 n-3$ & 0.54 & 0.17 & 0.27 & 0.54 \\
\hline $\mathrm{C} 22: 6 \mathrm{n}-3$ & 0.29 & 0.28 & 0.28 & 0.30 \\
\hline
\end{tabular}

${ }^{1} \mathrm{C}$, control feed; $\mathrm{L}$, control feed $+3 \%$ extruded linseed; LO, control feed $+3 \%$ extruded linseed $+0.6 \%$ oregano. ${ }^{2} \mathrm{NDF}$, neutral detergent fiber; $\mathrm{ADF}$, acid detergent fiber; ADL, acid detergent lignin

\section{Results}

The initial body weight of suckling kids was similar among dietary treatments (Table 3). The difference in final weight was reflected in a greater trend of weight gain of kids during trial. The LO diet had the lowest $(P>0.05)$ weight gains in comparison to the other treatments. The FCR resulted similar among dietary groups. Kids' slaughtering characteristics and meat cuts are given in Table 4. The three dietary groups performed quite equally for most carcass parameters and meat cuts evaluated. Both hot- and coldcarcass dressing (as percentage on body weight) in this research were similar among treatments (respectively: $P=$
Table 3: Effect of diet on in vivo performances of kids

\begin{tabular}{lllllll}
\hline Item & \multicolumn{3}{c}{ Diet } & SEM $^{2}$ & $P$-value \\
\cline { 2 - 5 } & $\mathrm{C}$ & $\mathrm{L}$ & $\mathrm{LO}$ & & \\
\hline Initial BW $(\mathrm{kg})$ & 3.36 & 3.24 & 3.37 & 0.356 & 0.911 \\
Final BW (kg) & 10.08 & 9.76 & 9.03 & 1.226 & 0.335 \\
Average daily BW gain (kg/d) & 111.9 & 108.9 & 94.2 & 9.587 & 0.051 \\
Average daily milk intake (kg/d) & 0.82 & 0.78 & 0.72 & 0.098 & 0.075 \\
Feed conversion ratio (kg/kg) & 7.32 & 7.17 & 7.64 & 0.202 & 0.061 \\
\hline C, control feed; L, control feed + 3\% extruded linseed; LO, control feed + $3 \%$ \\
extruded linseed + 0.6\% oregano. SEM: Standard error of means
\end{tabular}

Table 4: Slaughtering traits and meat cuts of kids

\begin{tabular}{llllll}
\hline Item & \multicolumn{5}{c}{ Diet } \\
\cline { 2 - 6 } & $\mathrm{C}$ & $\mathrm{L}$ & $\mathrm{LO}$ & $\mathrm{SEM}$ & $P$-value \\
\hline Slaughtering traits, kg & 10.08 & 9.76 & 9.03 & 2.009 & 0.063 \\
Slaughter weight & 2.56 & 2.30 & 2.07 & 1.014 & 0.456 \\
Right half carcass & 0.84 & 0.74 & 0.76 & 0.480 & 0.511 \\
Skin & 0.63 & 0.59 & 0.57 & 0.098 & 0.752 \\
Head & 0.48 & 0.51 & 0.65 & 0.033 & 0.101 \\
Empty GI tract & 0.74 & 0.58 & 0.59 & 0.074 & 0.095 \\
Offals & 67.95 & 69.15 & 68.32 & 0.885 & 0.057 \\
Hot carcass dressing, \% ${ }^{1}$ & 64.40 & 66.09 & 65.11 & 0.871 & 0.052 \\
Cold carcass dressing, \% ${ }^{1}$ & & & & & \\
Meat cuts, kg & 0.192 & 0.199 & 0.151 & 0.150 & 0.621 \\
Neck & 0.581 & 0.474 & 0.408 & 0.092 & 0.188 \\
Shoulder & 0.755 & 0.636 & 0.581 & 0.171 & 0.085 \\
Leg & 0.326 & 0.257 & 0.217 & 0.054 & 0.341 \\
Steaks & 0.134 & 0.142 & 0.129 & 0.079 & 0.436 \\
Loin & 0.265 & 0.209 & 0.220 & 0.086 & 0.638 \\
Brisket & C, control feed; L, control feed $+3 \%$ extruded linseed; LO, control feed + 3\% \\
extruded linseed + 0.6\% oregano. ${ }^{1} \%$ on body weight & & &
\end{tabular}

Table 5: Dissection data (\% on weight) of leg and loin of kids

\begin{tabular}{lllllll}
\hline Item & \multicolumn{7}{c}{ Diet } \\
\cline { 2 - 7 } & & $\mathrm{C}$ & $\mathrm{L}$ & $\mathrm{LO}$ & $\mathrm{SEM}$ & $P$-value \\
\hline Leg & $\mathrm{kg}$ & 0.755 & 0.636 & 0.581 & 0.071 & 0.085 \\
Lean & $\%$ & 61.07 & 61.55 & 62.85 & 0.652 & 0.226 \\
Dissectible fat & & $6.31 \mathrm{~b}$ & $4.50 \mathrm{a}$ & $6.13 \mathrm{~b}$ & 0.072 & $\mathbf{0 . 0 4 2}$ \\
Bone & & 32.63 & 33.95 & 31.02 & 1.233 & 0.055 \\
Loin & $\mathrm{kg}$ & 0.134 & 0.142 & 0.129 & 0.071 & 0.436 \\
Lean & $\%$ & 44.79 & 46.04 & 44.04 & 0.708 & 0.061 \\
Dissectible fat & & $13.97 \mathrm{~b}$ & $9.53 \mathrm{a}$ & $9.86 \mathrm{ab}$ & 2.066 & $\mathbf{0 . 0 3 9}$ \\
Bone & \multicolumn{7}{c}{41.24} & 44.43 & 46.10 & 1.014 & 0.062 \\
C, control feed; L, control feed $+3 \%$ extruded linseed; LO, control feed $+3 \%$ \\
extruded linseed + 0.6\% oregano; a-b: $P<0.05$
\end{tabular}

0.057 and $P=0.052$ ). No influence of feeding extruded linseed and oregano was observed on the main meat cuts and offal parts; however, the lean, fat and bone proportions of kids' leg and loin (Table 5) reported a lower percentage of dissectible fat in animals in extruded linseed group compared to the other two treatments (leg: $P=0.042$ and loin: $P=0.039$ ). In the present trial no difference was observed in L. lumborum and Semimembranosus meat muscles physical characteristics of suckling kids in terms of meat $\mathrm{pH}$ colour indices and WBS (Table 6), except for the meat cooking loss percentage that resulted significantly reduced $(P<0.05)$ in extruded linseed group for both meat muscles evaluated. Meat proximate composition of $L$. lumborum and Semimembranosus meat muscles was reported in Table 7, and from the obtained results, the kid 
Tarricone et al. / Intl J Agric Biol, Vol 25, No 5, 2021

Table 6: Meat characteristics from L. lumborum and Semimembranosus muscles

\begin{tabular}{|c|c|c|c|c|c|}
\hline \multirow[t]{2}{*}{ Item } & \multicolumn{3}{|c|}{ Diet } & \multirow[b]{2}{*}{ SEM } & \multirow[b]{2}{*}{$P$-value } \\
\hline & $\mathrm{C}$ & $\mathrm{L}$ & LO & & \\
\hline \multicolumn{6}{|l|}{ Longissimus lumborum } \\
\hline $\mathrm{pH}_{1}$ & 6.37 & 6.22 & 6.11 & 0.050 & 0.152 \\
\hline $\mathrm{pH}_{2}^{\#}$ & 5.88 & 5.79 & 5.54 & 0.088 & 0.223 \\
\hline$L^{*}$ & 47.27 & 46.38 & 44.50 & 0.747 & 0.061 \\
\hline$a^{*}$ & 5.20 & 4.98 & 5.55 & 0.369 & 0.059 \\
\hline$b^{*}$ & 12.52 & 10.89 & 10.63 & 0.971 & 0.063 \\
\hline WBS raw, $\mathrm{kg} / \mathrm{cm}^{2}$ & 2.40 & 2.24 & 2.30 & 0.092 & 0.369 \\
\hline WBS cooked, $\mathrm{kg} / \mathrm{cm}^{2}$ & 1.73 & 1.71 & 1.72 & 0.101 & 0.444 \\
\hline Cooking loss, $\%$ & $13.34 \mathrm{c}$ & $7.16 \mathrm{a}$ & $9.81 \mathrm{~b}$ & 2.215 & 0.030 \\
\hline \multicolumn{6}{|l|}{ Semimembranosus } \\
\hline $\mathrm{pH}_{1}$ & 6.55 & 6.39 & 6.05 & 0.074 & 0.113 \\
\hline $\mathrm{pH}_{2}{ }^{1}$ & 6.06 & 6.03 & 5.63 & 0.062 & 0.532 \\
\hline$L^{*}$ & 47.56 & 43.90 & 43.12 & 1.001 & 0.062 \\
\hline$a^{*}$ & 5.77 & 7.41 & 7.55 & 0.923 & 0.058 \\
\hline$b^{*}$ & 10.82 & 11.29 & 10.41 & 0.852 & 0.105 \\
\hline WBS raw, $\mathrm{kg} / \mathrm{cm}^{2}$ & 2.23 & 2.14 & 2.27 & 0.113 & 0.321 \\
\hline WBS cooked, $\mathrm{kg} / \mathrm{cm}^{2}$ & 1.76 & 1.79 & 1.81 & 0.092 & 0.465 \\
\hline Cooking loss, $\%$ & $16.06 \mathrm{~b}$ & $13.50 \mathrm{a}$ & $17.03 \mathrm{c}$ & 1.201 & 0.040 \\
\hline
\end{tabular}

Table 7: Chemical composition (\%) from Longissimus lumborum and Semimembranosus muscles

\begin{tabular}{llllll}
\hline Item & \multicolumn{5}{c}{ Diet } \\
\cline { 2 - 6 } & $\mathrm{C}$ & $\mathrm{L}$ & $\mathrm{LO}$ & $\mathrm{SEM}$ & $P$-value \\
\hline Longissimus lumborum & & & & & \\
Moisture & 74.05 & 75.53 & 75.13 & 0.110 & 0.114 \\
Protein & 21.25 & 20.05 & 20.53 & 0.352 & 0.070 \\
Lipid & 2.01 & 2.10 & 2.05 & 0.096 & 0.145 \\
Ash & 2.69 & 2.31 & 2.30 & 0.063 & 0.095 \\
Semimembranosus & & & & & \\
Moisture & 76.78 & 76.09 & 75.97 & 0.125 & 0.128 \\
Protein & 18.47 & 18.43 & 18.73 & 0.322 & 0.201 \\
Lipid & 1.93 & 1.82 & 1.77 & 0.049 & 0.087 \\
Ash & 2.81 & 3.65 & 3.53 & 0.052 & 0.062 \\
\hline C, control feed; L, control feed $+3 \%$ extruded linseed; LO, control feed $+3 \%$
\end{tabular}

extruded linseed $+0.6 \%$ oregano

meat nutritive value did not differ among dietary groups $(P$ $>0.05$ ) as previously observed by Jambrenghi et al. (2007). The Tables 8 and 9 report respectively the L. lumborum and Semimembranosus meat muscles fatty acid composition, being marginally influenced by the tested diet. As expected, however, kids' meat fatty acid profile was influenced by the nutritional characteristics of diets fed to goat. A significant increase $(P<0.05)$ with linseed and oregano (LO) inclusion in diet was found in L. lumborum meat for total SFA (palmitic acid) compared to the other two kid groups. Further, the same meat muscle had the lower content of EPA (C20:5 n3, eicosapentaenoic acid) and the high content of DPA (C22:5 n3, docosapentaenoic acid) in kids on $\mathrm{L}$ and LO treatments $(P<0.05)$. As given in Table 9 , a significant increase $(P<0.05)$ in oleic acid was detected in group linseed for Semimembranosus muscle, and in the same kids group a lower C20:1 n9 level was also detected. On the other hand, feeding experimental diets did not result in significant $(P>0.05)$ changes in total PUFA for both examined kid meat muscles.
Table 8: Fatty acid composition (\% total FA methyl esters) of kid meat from $L$. lumborum muscle

\begin{tabular}{llllll}
\hline Item & \multicolumn{5}{c}{ Diet } \\
\cline { 2 - 6 } & $\mathrm{C}$ & $\mathrm{L}$ & LO & SEM & $P$-value \\
\hline C10:0 & 0.11 & 0.16 & 0.23 & 0.031 & 0.256 \\
C12:0 & 0.52 & 0.60 & 0.65 & 0.101 & 0.574 \\
C14:0 & 5.46 & 5.74 & 6.95 & 0.955 & 0.095 \\
C15:0 & 0.35 & 0.31 & 0.39 & 0.042 & 0.464 \\
C16:0 & 23.04 & 22.95 & 24.89 & 1.147 & 0.075 \\
C17:0 & 0.51 & 0.48 & 0.56 & 0.098 & 0.297 \\
C18:0 & 11.88 & 12.40 & 11.41 & 1.023 & 0.063 \\
C20:0 & 0.05 & 0.04 & 0.06 & 0.001 & 0.355 \\
C21:0 & 0.05 & 0.04 & 0.05 & 0.002 & 0.369 \\
C22:0 & 0.34 & 0.26 & 0.27 & 0.040 & 0.222 \\
C23:0 & 0.89 & 0.93 & 0.79 & 0.225 & 0.188 \\
CSFA & 43.18 a & 43.91 a & 46.27 b & 1369 & $\mathbf{0 . 0 4 3}$ \\
C14:1 & 0.05 & 0.12 & 0.19 & 0.005 & 0.396 \\
C15:1 & 0.18 & 0.19 & 0.25 & 0.006 & 0.299 \\
C16:1 trans & 0.38 & 0.39 & 0.44 & 0.098 & 0.458 \\
C16:1 cis & 1.68 & 1.43 & 1.89 & 0.274 & 0.366 \\
C17:1 & 0.40 & 0.32 & 0.36 & 0.052 & 0.501 \\
C18:1 n9 trans & 0.15 & 0.23 & 0.14 & 0.010 & 0.133 \\
C18:1 n9 cis & 34.11 & 34.21 & 32.84 & 1.526 & 0.077 \\
C20:1 n9 & 0.92 & 0.46 & 0.60 & 0.195 & 0.085 \\
C22:1 n9 & 3.69 & 2.65 & 2.76 & 0.210 & 0.057 \\
\& MUFA & 41.56 & 40.01 & 39.47 & 1.035 & 0.059 \\
C18:2 n6 trans & 0.11 & 0.09 & 0.36 & 0.015 & 0.113 \\
C18:2 n6 cis & 8.48 & 7.23 & 7.47 & 1.114 & 0.071 \\
C18:3n6 & 1.15 & 1.27 & 1.10 & 0.206 & 0.256 \\
C18:3n3 & 0.10 & 0.18 & 0.09 & 0.003 & 0.118 \\
C20:2 n6 & 0.54 & 0.44 & 0.47 & 0.062 & 0.254 \\
C20:3 n6 & 4.88 & 4.96 & 4.75 & 0.369 & 0.137 \\
C20:4 n6 ARA & 0.03 & 0.08 & 0.06 & 0.003 & 0.266 \\
C20:3 n3 & 0.10 & 0.04 & 0.06 & 0.003 & 0.341 \\
C22:2n6 & 0.16 & 0.10 & 0.10 & 0.010 & 0.478 \\
C20:5 n3 EPA & $1.01 \mathrm{~b}$ & 0.42 a & 0.51 a & 0.104 & $\mathbf{0 . 0 3 3}$ \\
C22:5 n3 DPA & 0.53 b & 0.89 a & 0.90 a & 0.147 & $\mathbf{0 . 0 4 0}$ \\
C22:6 n3 DHA & 0.59 & 0.60 & 0.64 & 0.071 & 0.115 \\
\& PUFA & 17.67 & 16.29 & 16.51 & 0.804 & 0.060 \\
\hline C, control feed; L, control feed + 3\% extruded linseed; LO, control feed + 3\% extruded \\
linseed + 0.6\% oregano. EPA: eicosapentaenoic acid; DPA: docosapentaenoic acid \\
docosapentaenoic acid; DHA: docosahexaenoic acid; a-b: P $<0.05$ & \\
& & & & &
\end{tabular}

\section{Discussion}

The productivity of small ruminants, in particular kids, in many countries is quite reduced and it resulted mainly related to nutrition, breed and rearing conditions. The limits of goats and kids nutrition in Mediterranean regions can be partially correlated to the environmental conditions influencing feed availability and pasture qualitative characteristics.

In our research, suckling kids did not vary among diets, and these differences are also reflected only in a greater trend of body gains. The findings observed in present study are consistent with those previously found by Giannico et al. (2009) and more recently by Rotondi et al. (2018), who fed respectively lambs and kids with diets containing extruded linseed and oregano as feed supplement. Further, other previous researchers confirmed that extruded linseed can be an effective ingredient in goat concentrate with no adverse effect on suckling kids or lambs' performance compared to control diet (Colonna et 
Table 9: Fatty acid composition (\% total FA methyl esters) of kid meat from Semimembranosus muscle

\begin{tabular}{|c|c|c|c|c|c|}
\hline \multirow[t]{2}{*}{ Item } & \multicolumn{5}{|c|}{ Diet } \\
\hline & $\mathrm{C}$ & $\mathrm{L}$ & LO & SEM & $P$-value \\
\hline C10:0 & 0.09 & 0.17 & 0.17 & 0.009 & 0.158 \\
\hline C12:0 & 0.45 & 0.64 & 0.68 & 0.287 & 0.202 \\
\hline C14:0 & 4.67 & 5.83 & 6.70 & 0.801 & 0.064 \\
\hline C15:0 & 0.27 & 0.34 & 0.37 & 0.150 & 0.096 \\
\hline C16:0 & 22.16 & 22.76 & 24.15 & 1.114 & 0.060 \\
\hline $\mathrm{C} 17: 0$ & 0.45 & 0.55 & 0.55 & 0.236 & 0.147 \\
\hline C18:0 & 12.66 & 12.14 & 12.03 & 1.098 & 0.212 \\
\hline C20:0 & 0.04 & 0.05 & 0.06 & 0.002 & 0.696 \\
\hline C21:0 & 0.06 & 0.05 & 0.04 & 0.002 & 0.854 \\
\hline C22:0 & 0.42 & 0.29 & 0.31 & 0.111 & 0.322 \\
\hline C23:0 & 1.13 & 0.98 & 0.93 & 0.165 & 0.165 \\
\hline$\sum \mathrm{SFA}$ & 42.39 & 43.80 & 45.99 & 1.152 & 0.052 \\
\hline$\overline{\mathrm{C}} 14: 1$ & 0.04 & 0.17 & 0.14 & 0.008 & 0.095 \\
\hline C15:1 & 0.16 & 0.21 & 0.24 & 0.085 & 0.177 \\
\hline C16:1 trans & 0.32 & 0.44 & 0.41 & 0.049 & 0.369 \\
\hline C16:1 cis & 1.37 & 1.51 & 1.52 & 0.174 & 0.828 \\
\hline $\mathrm{C} 17: 1$ & 0.28 & 0.43 & 0.19 & 0.033 & 0.125 \\
\hline $\mathrm{C} 18: 1 \mathrm{n} 9$ trans & 0.13 & 0.24 & 0.13 & 0.010 & 0.163 \\
\hline C18:1 n9 cis & $31.98 \mathrm{~b}$ & $34.69 a$ & $31.55 \mathrm{~b}$ & 2.005 & 0.029 \\
\hline $\mathrm{C} 20: 1 \mathrm{n} 9$ & $1.08 \mathrm{~b}$ & $0.57 \mathrm{ab}$ & $0.32 \mathrm{a}$ & 0.099 & 0.038 \\
\hline $\mathrm{C} 22: 1 \mathrm{n} 9$ & 4.78 & 3.42 & 3.32 & 0.552 & 0.059 \\
\hline$\sum$ MUFA & $40.12 a b$ & $41.67 \mathrm{a}$ & $37.83 \mathrm{~b}$ & 0.715 & 0.042 \\
\hline $\mathrm{C} 18: 2 \mathrm{n} 6$ trans & 0.12 & 0.18 & 0.12 & 0.006 & 0.774 \\
\hline $\mathrm{C} 18: 2 \mathrm{n} 6$ cis & 7.88 & 7.64 & 8.66 & 0.566 & 0.146 \\
\hline C18:3n6 & 1.17 & 1.24 & 1.12 & 0.074 & 0.363 \\
\hline C18:3n3 & 0.11 & 0.18 & 0.17 & 0.009 & 0.622 \\
\hline C20:2 n6 & 0.70 & 0.47 & 0.32 & 0.022 & 0.179 \\
\hline C20:3 n6 & 4.21 & 4.17 & 4.22 & 0.061 & 0.696 \\
\hline C20:4 n6 ARA & 0.02 & 0.03 & 0.02 & 0.001 & 0.785 \\
\hline $\mathrm{C} 20: 3 \mathrm{n} 3$ & 0.13 & 0.08 & 0.06 & 0.005 & 0.444 \\
\hline $\mathrm{C} 22: 2 \mathrm{n} 6$ & 0.20 & 0.12 & 0.14 & 0.013 & 0.312 \\
\hline C20:5 n3 EPA & 1.12 & 0.67 & 0.35 & 0.101 & 0.058 \\
\hline C22:5 n3 DPA & 0.66 & 1.01 & 1.14 & 0.040 & 0.061 \\
\hline C22:6 n3 DHA & 0.67 & 0.70 & 0.56 & 0.022 & 0.254 \\
\hline$\sum$ PUFA & 16.99 & 16.49 & 16.88 & 0.113 & 0.102 \\
\hline
\end{tabular}

al. 2011; Rotondi et al. 2018).

The slaughtering characteristics and meat cuts of suckling kids under the three treatments performed similarly for most of the parameters examined. However, the observed findings resulted quite lower than those assessed in previous reports using different goat breed in Italy (Rotondi et al. 2018; Colonna et al. 2020), and this might be mainly due to feeding practice and management as well as related to the composition of goat concentrate diet. Moreover, other studies evaluating different natural dietary supplements reported inconstant findings regarding the carcass parameters. In the current study no significant differences were observed in $L$. lumborum and Semimembranosus meat muscles physical characteristics of suckling kids, even if it was found that meat cooking loss percentage that resulted $\mathrm{s}$ reduced in suckling kids in extruded linseed group. Unfortunately, there are no available reports on the effects of extruded linseed and oregano supplementation on suckling kid meat physical characteristics. In particular, among other meat-producing livestock species, it observed a similar trend in meat from lambs (Colonna et al. 2011), bulls (Ragni et al. 2014; Tarricone et al. 2019a) and pigs (Tarricone et al. 2019b). Nevertheless, the variations between our results and the findings observed in previous trials can be mainly due to the difference in using other animal breeds, feedstuffs, and concentrate characteristics (Toteda et al. 2011; Tufarelli et al. 2013). In the examined meat muscles L. lumborum and Semimembranosus) of suckling Grigia di Potenza kids, the dietary treatments did not influence the proximate nutritional composition of meat, and this trend was also observed by Jambrenghi et al. (2007) in suckling kids. In our feeding trial, the FA profile of both meat muscles were to some extent influenced by the dietary treatments; however, as expected, the kids' meat FA profile varied based on the nutritional characteristics of diets fed to goat. A increase with linseed plus oregano in diet was found in $L$. lumborum meat for total SFA (palmitic acid) compared to the other two kid groups. Further, a significant increase in oleic acid was detected in linseed group for Semimembranosus muscle. At this regard, the main monounsaturated fatty acid in meat muscle was the oleic acid, which might be linked to the high concentration in linseed oil, as previously demonstrated by Colonna et al. (2011) and Rotondi et al. (2018). The high level of oleic acid in diet including extruded linseed determined higher concentration of this fatty acid in the meat of suckling kids fed the tested high-quality feed ingredient than those fed the other diets. Also, there were no dietary effects on the total PUFA (polyunsaturated fatty acid) in both kid meat cuts, and this was in accordance with the results of Berthelot $e t$ al. (2010) in meat from lambs.

\section{Conclusion}

Based on the findings, the present study confirms that both extruded linseed and oregano can be added in goat diet as no decrease in growth traits and meat quality was assessed in suckling kids, being also capable to positively modulate meat lipid profile. Therefore, this experiment therefore supported the idea that including extruded linseed and oregano in the feeding system of lactating goat would produce kid meat with a healthier lipid profile with no detrimental effect on growth performance.

\section{Acknowledgements}

The present study was founded and supported by the Region Basilicata (Italy) "VAL.BI.OVI.CAP. - PSR Region Basilicata 2014-2020 - M 10 - Sub M 10.2 - Support for the sustainable conservation, use and development of genetic resources in agriculture".

\section{Author Contributions}

All authors have equally contributed to the manuscript and 
read and approved the content of the manuscript.

\section{Conflicts of Interest}

All other authors declare no conflicts of interest

\section{Data Availability}

Data presented in this study are available on fair request to the corresponding author.

\section{Ethics Approval}

Ethical review and approval were waived for this study, due to animals were cared for and handled in compliance with the EU legislation on animal welfare regulations (Directive 2010/63/EU which updates and replaces the 1986 Directive 86/609/EEC on the protection of animals used for scientific purposes) and following the University research policy.

\section{References}

AOAC (2000). Official Methods of Analysis, $17^{\text {th }}$ Edition. Association of Official Analytical Chemists, Arlington, Virginia, USA

Berthelot V, P Bas, P Schmidely (2010). Utilization of extruded linseed to modify fatty composition of intensively-reared lamb meat: Effect of associated cereals (wheat vs. corn) and linoleic acid content of the diet. Meat Sci 84:114-124

Cimmino R, CMA Barone, S Claps, E Varricchio, D Rufrano, M Caroprese, M Albenzio, P De Palo, G Campanile, G Neglia (2018). Effects of dietary supplementation with polyphenols on meat quality in Saanen goat kids. BMC Vet Res 14; Article 181

Colonna MA, P Rotondi, M Selvaggi, AC Jambrenghi, M Ragni, S Tarricone (2020). Sustainable rearing for kid meat production in Southern Italy marginal areas: a comparison among three genotypes. Sustainability 12; Article 6922

Colonna MA, F Giannico, A Coluccia, GD Bello, G Vonghia, AC Jambrenghi (2011). Dietary supplementation with extruded linseed and linseed oil in lamb feeding: productive performances and meat quality traits. Prog Nutr 13:111-124

Facciolongo AM, A Lestingi, MA Colonna, F Nicastro, DD Marzo, F Toteda (2018). Effect of diet lipid source (linseed versus soybean) and gender on performance, meat quality and intramuscular fatty acid composition in fattening lambs. Small Rumin Res 15:11-17

Folch J, M Lees, GH Sloan-Stanley (1957). A simple method for the isolation and purification of total lipids from animal tissues. $J$ Biol Chem 226:497-509

Giannico F, MA Colonna, A Coluccia, D Crocco, G Vonghia, C Cocca, A Caputi Jambrenghi (2009). Extruded linseed and linseed oil as alternative to soybean meal and soybean oil in diets for fattening lambs. Ital J Anim Sci 8:495-497
Jambrenghi AC, MA Colonna, F Giannico, G Cappiello, G Vonghia (2007). Effect of goat production systems on meat quality and conjugated linoleic acid(CLA) content in suckling kids. Ital J Anim Sci 6:612-614

Lunn J, HE Theobald (2006). The health effects of dietary unsaturated fatty acids. Nutr Bull 31:178-224

Naveena BM, AR Sen, S Vaithiyanathan, Y Babji, N Kondaiah (2016) Comparative efficacy of pomegranate juice, pomegranate rind powder extract and BTH as antioxidants in cooked chicken patties. Meat Sci 120:107-117

NRC (2007) Nutrient Requirements of Small Ruminants. National Academy Press, Washington DC, USA

Ragni M, V Tufarelli, F Pinto, F Giannico, V Laudadio, A Vicenti, MA Colonna (2015). Effect of dietary safflower cake (Carthamus tinctorius $\mathrm{L}$.) on growth performances, carcass composition and meat quality traits in Garganica breed kids. Pak J Zool 47:193-199

Ragni M, F Toteda, V Tufarelli, V Laudadio, A Facciolongo, F Dipalo, A Vicenti (2014). Feeding of extruded flaxseed (Linum usitatissimum L.) and pasture in Podolica young bulls: Effects on growth traits, meat quality and fatty acid composition. Pak J Zool 46:1101-1109

Rotondi P, MA Colonna, G Marsico, F Giannico, M Ragni, AM Facciolongo (2018). Dietary supplementation with oregano and linseed in Garganica suckling kids: Effects on growth performances and meat quality. Pak J Zool 50:1421-1433

SAS (2004). SAS/STAT 9.13 User's Guide. Statistical Analysis System Inst, Cary, North Carolina, USA

Sarvar EN, MM Moeini, M Poyanmehr, E Mikaeli (2009). The effects of docking on growth traits, carcass characteristics and blood biochemical parameters of Sanjabi fat-tailed lambs. Asian-Aust $J$ Anim Sci 22:796-802

Selvaggi M, V Tufarelli (2012). Caseins of goat and sheep milk: analytical and technological aspects. In: Casein: Production, Uses and Health Effects, pp: 1-25. Ventimiglia AM, JM Birkenhäger (Eds.). Nova Science Publishers, New York, USA

Šicklep M, M Čandek-Potokar (2007). Pork color measurement as affected by bloom time and measurement location. J Musc Foods 18:78-87

Simitzis PE, SG Deligeorgis, JA Bizelis, A Dardamani, I Theodosiou, K Fegeros (2008). Effect of dietary oregano oil supplementation on lamb meat characteristics. Meat Sci 79:217-223

Tarricone S, MA Colonna, F Giannico, AM Facciolongo, A Caputi Jambrenghi, M Ragni (2019a). Effects of dietary extruded linseed (Linum usitatissimum L.) on performance and meat quality in Podolian young bulls. S Afr J Anim Sci 49:781-789

Tarricone S, MA Colonna, F Giannico, M Ragni, A Lestingi, AM Facciolongo (2019b). Effect of an extruded linseed diet on meat quality traits in Nero Lucano pigs. S Afr J Anim Sci 49:1093-1103

Toteda F, A Facciolongo, M Ragni, A Vicenti (2011). Effect of suckling type and PUFA use on productive performances, quanti-qualitative characteristics of meat and fatty acid profile in lamb. Prog Nutr 13:125-134

Tufarelli V, E Casalino, AG D'Alessandro, V Laudadio (2017). Dietary phenolic compounds: Biochemistry, metabolism and significance in animal and human health. Curr Drug Metabol 18:905-913

Tufarelli V, M Introna, E Cazzato, D Mazzei, V Laudadio (2013). Suitability of partly destoned exhausted olive cake as by-product feed ingredient for lamb production. J Anim Sci 91:872-877

Tufarelli V, RU Khan, V Laudadio (2011). Feeding of wheat middlings in lamb total mixed rations: effects on growth performance and carcass traits. Anim Feed Sci Technol 170:130-135 